\title{
Técnicas de análisis multivariado, modelamiento factorial múltiple y PLS-PATH para estudio y clasificación de tipos de mieles venezolanas de los estados de Lara y Yaracuy
}

\author{
Multivariate techniques, factorial multiple analysis and PLS-PATH \\ modeling for study and classification of venezuelan honeys from states \\ of Lara and Yaracuy
}

Recepción: 3 de junio de 2016

Aceptación: 29 de diciembre de 2016

\begin{abstract}
Honey is a natural product made mainly from the nectar of flowers and their final quality depend on their botanical and geographical origin. Its variability is large even for honey produce at the same area. The aim of this work have been centered in evaluation of Venezuelan honey from Lara and Yaracuy States, taking as a reference the physicochemical parameters of color, chromaticity of CIELab, $\mathrm{pH}$, free acidity $\left(\mathrm{X}_{\mathrm{AL}}\right)$, total sugars $(X s)$, moisture $(X w)$, ash $(\mathrm{Xc})$, electrical conductivity $(\mathrm{EC})$, density, and water activity $\left(a_{w}\right)$. Additionally have been associated the sensory attributes and intrinsic properties of honey for a classification process for homogeneous honey using multivariate analysis, multiple factor and PLSPATH. The relationship between the answers and regression, reveals types of honey with $50 \%$ dominantly amber (A), $40 \%$ amber light (LA) and $10 \%$ dark amber (DA) of multiflower nature. The physicochemical properties and sensory attributes are conducive to differentiation in accordance to their geographical origin.
\end{abstract}

Keywords: Multivariate analysis. Classification methods. Venezuelan honeys. Physicochemical properties. PLS-PATH.

\section{Resumen}

La miel es un producto natural elaborado principalmente a partir del néctar de las flores, su calidad final esta en función de su origen botánico y geográfico y su variabilidad es amplia inclusive para mieles de la misma región. En éste trabajo se han evaluado y clasificado tipos de venezolanas de los estados Lara y Yaracuy, tomando como referencia los parámetros fisicoquímicos de color, cromaticidad del sistema CIELab, $\mathrm{pH}$, acidez libre $\left(\mathrm{X}_{\mathrm{AL}}\right)$, azucares totales $(X s)$, humedad $(X w)$, cenizas $(\mathrm{Xc})$; conductividad eléctrica (CE), densidad y actividad de agua $\left(a_{w}\right)$. Adicionalmente se han relacionado los atributos sensoriales y propiedades intrínsecas de las mieles para el proceso de clasificación de mieles homogéneas a partir del análisis multivariado, factorial múltiple y PLS-PATH. La relación entre las respuestas y la regresión, revela tipos de mieles con $50 \%$ dominantemente ámbar (A), $40 \%$ ámbar claro (LA) y $10 \%$ ámbar obscura (DA) de naturaleza multifloral. Las propiedades fisicoquímicas y atributos sensoriales son conducentes a la diferenciación en virtud a su origen geográfico.

Palabras clave: Análisis multivariado. Métodos de clasificación. Mieles venezolanas. Propiedades fisicoquímicas. PLS-PATH.

\footnotetext{
Grupo de Investigaciones Mellitopalinológicas y Propiedades Fisicoquímicas de Alimentos. Universidad del Tolima. Colombia.

Autor de Corrrespondencia: mposorio@ut.edu.co

Departamento de Matemáticas y Estadística. Facultad de Ciencias. Universidad del Tolima. Colombia.

Correo electrónico: nrodriguezar@gmail.com

Departamento de Química. Facultad de Ciencias. Universidad del Tolima. Colombia.

Correo electrónico: lipfa@ut.edu.co
} 


\section{Introducción}

La Organización Mundial para la Alimentación, ha definido a la miel como una sustancia dulce elaborada por la abeja (Apis mellifera L.) y sus diferentes subespecies, a partir del néctar de las flores y de otras secreciones extraflorales, que las abejas liban, transportan, deshidratan, concentran y alma- cenan en los panales [1-3]. Debido a que la miel hereda las propiedades de las plantas, su color, aroma, sabor y propiedades fisicoquímicas dependen de las flores utilizadas por las abejas, aunque las condiciones climáticas también influyen en la composición y sus propiedades [4]. La demanda de miel entre los consumidores a nivel mundial ha crecido de manera significativa, haciéndose necesaria una verificación de su autenticidad, con el objetivo de satisfacer los requerimientos de los consumidores, que apetecen productos orgánicos, sanos y diferenciados, elaborados bajo rigurosos estándares de calidad y con la certificación de organizaciones y autoridades responsables de la vigilancia y control [5-8].

El origen botánico y geográfico de la miel sigue siendo difícil de determinar, por ello se hace cada vez más importante estandarizar protocolos de análisis que contribuyan a la verificación de la calidad de éste importante producto de la colmena e identificar su origen floral para reducir el mercado de mieles fraudulentas y suplantadas. Debido a que la miel hereda de las plantas con flores su color, aroma, sabor y propiedades fisicoquímicas, las condiciones pedoclimáticas también influyen en la composición de las mismas. El interés en el análisis de la miel se ha incrementado con el objetivo de satisfacer las regulaciones solicitadas por las diferentes autoridades, particularmente en la miel de origen monofloral [9].

Las miel es tan diversa como lo es su origen botánico, su naturaleza, composición y distintas tonalidades de color; éste último cambia en función de la presencia de pigmentos, carotenos y xantófilas, de los derivados fenólicos, presencia de policétidos, terpenos, esteroides y alcaloides, entre otros [10]. El color de la miel es uno de los atributos más variables y está determinado principalmente por su origen botánico, pero también depende del contenido de cenizas, la temperatura y tiempo de almacenamiento. A pesar de la gran importancia de éste parámetro aún no existe un método aceptado internacionalmente para su determinación. Los métodos más usados se basan en la comparación óptica, utilizando el color de clasificación simple. Durante muchos años, el estándar de referencia en EE.UU, ha sido el clasificador Pfund, que proporciona una medida de la intensidad del color a lo largo de tonalidades ambar en la miel [11-14].

Las mieles florales generalmente son de color ámbar, aunque también se conocen productos oscuros, como ocurre con las mieles de mielada de Roble (Quercus sp.) y Ulmo (Quillaja saponaria); también se ha atribuido el color al contenido mineral de las muestras y a la presencia de tirosina y triptófano, ausentes por lo general en mieles claras [15]. La calidad y tonalidades de color de distintos tipos de miel está supeditada a los periodos estacionales [16]. La aplicación de los métodos espectrofotométricos por medición de la transmitancia y/o la reflectancia en mieles se ha ilustrado a partir de los trabajos de varios investigadores [6, 15-18].

La determinación de los parámetros de color utilizando el CIE L* $a^{*} b^{*}$, método triestímulo también se ha empleado [19-20], pero no se ha introducido en la rutina para análisis de miel. Sin embargo, antes de que estas técnicas se puedan aplicar a la clasificación, tienen que ser validados con los métodos clásicos de clasificación de color [12]. El color es la primera propiedad sensorial percibida por los consumidores, lo que determina la compra de la misma. A pesar de que existe poca información acerca de la aceptabilidad de los consumidores por este parámetro visible [12], las mieles de mielada oscuras, en Alemania, Austria y Suiza, son especialmente apreciadas. El Mapeo de preferencias del consumidor es una herramienta comúnmente utilizada en la comprensión de los atributos sensoriales [11, 21-24].

La evaluación sensorial de los alimentos es una disciplina integrada que permite establecer la calidad de los productos sobre la base de sus atributos. El control y calidad final de los alimentos es una tarea que demanda vigilancia permanente de las materias primas, de procesos y producto terminado. En el caso de los productos con denominación de origen como es el de la miel, éstos controles toman vigor pues el producto es de alto valor comercial y lleva además implícita la declaratoria de autenticidad. La creciente necesidad de gestión de calidad en industrias de alimentos, ha permitido que se construyan sistemas con toma de decisiones automatizadas para la evaluación de productos. La gestión de calidad por su parte conlleva en la actualidad a la implementación de métodos que permitan clasificar grupos de alimentos con propiedades específicas similares teniendo en cuenta la marca, el origen geográfico, su autenticidad o adulteración [25]. 
En los métodos de clasificación los productos se identifican y agrupan en conjuntos finitos de clase, comparando las propiedades y atributos intrínsecos. Existe una amplia variedad de enfoques de las técnicas de reconocimiento, en éste sentido son bien conocidas las técnicas de análisis multivariado, como el análisis discriminante lineal (AD), análisis discriminante con mínimos cuadrados parciales (AD-PLS), árboles de clasificación y regresión (ACR), sistema de vectores soporte (SVS) y redes neuronales artificiales (RNA). Actualmente, es posible generar matrices de composición tomando como referencia el origen, la naturaleza del producto y su atributos sensoriales, posibilitando la generación de bases de datos que permitan realizar discriminaciones, mediante análisis exploratorios y técnicas de clasificación, sin embargo el advenimiento de nuevas metodologías de análisis estadístico, han hecho posible el desarrollo de procesos robustos [26-29]. Las aplicaciones y uso de técnicas de análisis discriminante por su parte se fundamentan en extraer a partir de $x_{1}, \ldots, x_{p}$ variables observadas en $k$ grupos, $m$ funciones $y_{1}, \ldots, y_{m}$ que conducen a la generación de funciones de modo que las combinaciones lineales proporcionen la mayor discriminación posible entre los grupos.

Las técnicas de Análisis Factorial Múltiple (AFM), ha incursionado de manera importante en la actualidad, esta combina el análisis de componentes principales (CP) y el de correlación canónico. Los métodos tradicionales por (CP), Análisis Factorial de Correspondencias (AFC) y Análisis de Correspondencias Múltiples, (ACM), sólo permiten estudiar las relaciones entre las variables de la misma naturaleza, todas cuantitativas o todas cualitativas, lo que restringe su utilización. En ocasiones, es posible recoger gran cantidad de información que es interesante relacionar, aunque mida aspectos heterogéneos. Esta información puede ser estructurada en diferentes grupos y su análisis mediante (AFM), proporciona una visión más rica del fenómeno a estudiar. AFM, se basa en la metodología de Análisis de Componentes Principales y actúa en dos etapas.

En la construcción de modelos PLS, se acude al uso de la matriz de datos $\mathrm{X}$, que contienen las muestras $n$ y las características descriptoras $p$, del orden $n \times p$, encontrándose problemas de multicolinealidad y alta dimensionalidad de las variables predictoras. La metodología PLS, combina características del análisis de componentes principales ACP y análisis de regresión múltiple ARM; en PLS, a diferencia del ACP, se requiere de los datos de entrada de la matriz de predictoras $\mathrm{X}$ y la de respuestas $\mathrm{Y}$, de orden $n \times p$ [30-32]. La matriz de datos es $X, I$ el set de datos individuales, $K$ las variables (incluyendo todos los grupos) o el set de índices de las variables considerados, $J$ es el grupo de variables, $K_{j}$ es el set de variables en el grupo $j$ o el set de índice de variables en el grupo $j, X_{j}$ la subtabla asociada con el grupo $j[33]$.

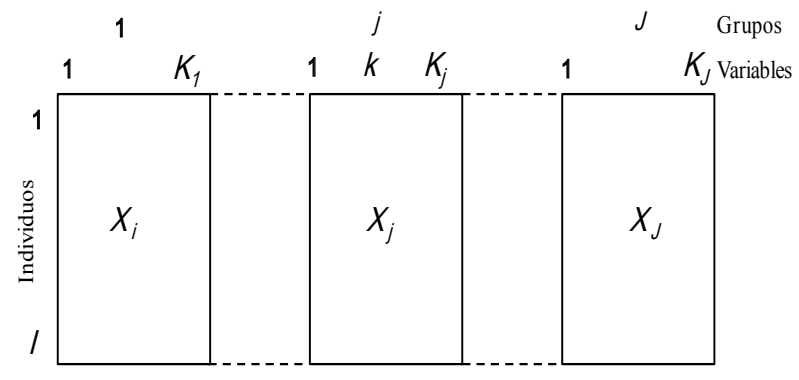

Figura 1. Relación estructural de grupos de componentes e individuos del análisis factorial múltiple (AFM).

Elempleo y uso de los modelos de redes neuronales se han implementado para demostrar el rendimiento y exactitud de las técnicas convencionales de clasificación estadística en muchas aplicaciones [34]. La red se usa estableciendo varios patrones de entrada y un patrón o característica de salida (Figura, 2). En el análisis de clasificación, los datos ingresan por medio de la "capa de entrada", pasan a través de la capa oculta salen por la "capa de salida", la capa oculta puede estar constituida por varias capas. Estos modelos son capaces de identificar semejanzas entre los patrones de entrada causando su disgregación en grupos del parámetro de salida $[35,36]$.

Desde el punto de vista de las RNA, se debe ajustar a un sistema de cálculo, teniendo en cuenta un vector de entradas que conducen a las respuestas de salida (Figura 2), [35-37]. El conjunto del sistema esta constituido por las entradas $x_{j}(t)$ y de procesos sinápticos $w_{i j}$, una regla de propagación, donde $h_{i}(t)$ $=s\left(w_{i j}, x_{j}(t)\right.$ y una función de activación $y_{i}(t)=f_{i}$ $\left(y_{i}(t-1), h_{i}(t)\right.$, que proporciona su salida $y_{i}(t)$. Las entradas y salidas de las RNA pueden ser binarias (digitales) o continuas (analógicas), dependiendo del modelo y de la aplicación. En procesos de clasificación se emplearían salidas digitales $(0,+1)$, mientras que en un problema de ajuste funcional de una aplicación multivariable continua, se emplearían salidas continuas dentro de un cierto intervalo. La regla de propagación, a partir de las entradas de la neurona y de sus pesos proporciona el valor del potencial postsináptico. La regla más habitual es lineal, llevando a cabo la suma de las entradas 
ponderada con los pesos sinápticos. A esta expresión se suele añadir un parámetro adicional $i$ denominado umbral [38-40].

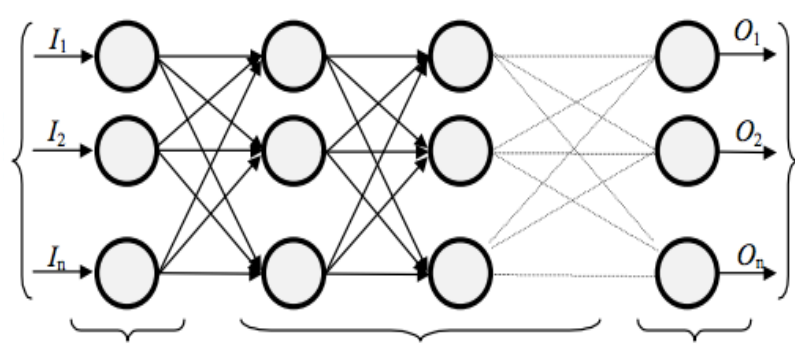

Figura 2. Representación general de una red neuronal artificial como estructura de clasificación.

El análisis sensorial (AS), se refiere a la medición y cuantificación de las características de los productos alimenticios evaluables por los sentidos. La correlación de las propiedades fisicoquímicas de los alimentos y los resultados de las evaluaciones sensoriales realizadas en paneles de valoración sensorial, por si mismas constituyen un reto al análisis estadístico. El (AS) de las mieles se consolida como una herramienta singular y de complemento en la determinación de su origen botánico [7, 18, 41-43].

En Venezuela los trabajos de caracterización e identificación de la calidad de mieles han sido realizados por diversos investigadores, en diferentes localidades de los estados Táchira, Mérida y Zulia [44-45], pero más recientemente, se reportó un trabajo ampliado a la calidad de las mieles del estado Zulia [45] y los perfiles sensoriales de mieles de los estados de Lara y Yaracuy [46] que es complementario al trabajo de caracterización reportado por Piana y colaboradores [47]. Muestras de los estados de Barinas, Cojedes, Anzoátegui, Bolívar, Lara fueron estudiados por Swanson y Lewis [44] y se ha relacionado la actividad antibacteriana como concentración mínima inhibitoria frente a $S$. aureus y E. coli, capacidad antioxidante, la actividad diastásica entre otros parámetros físicoquímicos que revelan la calidad de las mieles venezolanas. El contenido mineral igualmente ha sido reportado en muestras del Zulia [45].

Los trabajos orientativos al estudio de mieles y productos de la colmena en relación a los métodos de clasificación aún son reducidos. Los estudios en mieles se han basado en la determinación de sus propiedades químicas, físicas o biológicas. Varios estudios han intentado establecer rangos adecuados considerando algunas de sus propiedades y origen botánico mediante diferentes técnicas de análisis [23, $37,49]$. En éste trabajo se ha planteado el estudio de algunas de las propiedades fisicoquímicas y atributos sensoriales de mieles tropicales venezolanas de los estados de Lara y Yaracuy, tomando como referencia técnicas modernas de clasificación multivariado, haciendo uso de sistemas de análisis de conglomerados, redes neuronales, métodos discriminantes, modelamiento factorial múltiple y PLS-PATH, previa evaluación de sus propiedades fisicoquímicas y atributos intrínsecos de color y cromaticidad.

\section{Materiales y métodos}

\subsection{Zona de estudio}

Se realizaron desplazamiento a las principales zonas de producción, de los Estados Lara y Yaracuy (R.B Venezuela, Figura 1). En éstas se aprecian las zonas de vida del sistema de clasificación de Holdridge 1987, de bosque seco y muy seco tropical (bs-T; $b m s-T)$ y el bosque seco y muy seco premontano, (bs- $P M$; $b m s-P M)$ y que alterna con bosque húmedo premontano (bh-PM). En el estado Lara predominan condiciones de ( $b s-T$; $b m s-T, b s-$ $P M$; $b m s-P M)$, que alterna con sistemas de ( $b h-P M)$, representa el 4.8 del área total $19,800 \mathrm{~km}^{2}$. En la mayor parte del estado la evaporación supera a las precipitaciones, estas son del orden de $650 \mathrm{~mm}$ /año. Los índices pluviométricos diversos según el área y la temperatura media está entre 22 y $28^{\circ} \mathrm{C}$, con una media de $23,8^{\circ} \mathrm{C}$. El estado está constituido por 9 municipios y 58 parroquias. La sección geográfica que comprende el estado de Yaracuy, reúne 14 municipios. En algunos aspectos la vegetación es similar a la de Lara. No obstante se presentan reductos propios de bosque y de galería en las márgenes de los ríos Aroa y Yaracuy, también aparecen formaciones xerófilas. El clima en las cumbres de sus montañas y subtropical, en los valles altos de la Sierra de Nirgua. En la mayor parte del estado, impera el clima tropical, pero se pueden presentar hasta tres tipos de climas, bien definidos, dependiendo de la altura. Los dos estados forman parte de la transición desde los Andes a zona marítimas costera, en la depresión Barquisimeto-Carora.

\subsection{Muestras}

La recolección de las mieles se realizó en apiarios establecidos en zonas apícolas de los estados de Lara y Yaracuy de la región centro occidental del país, en 


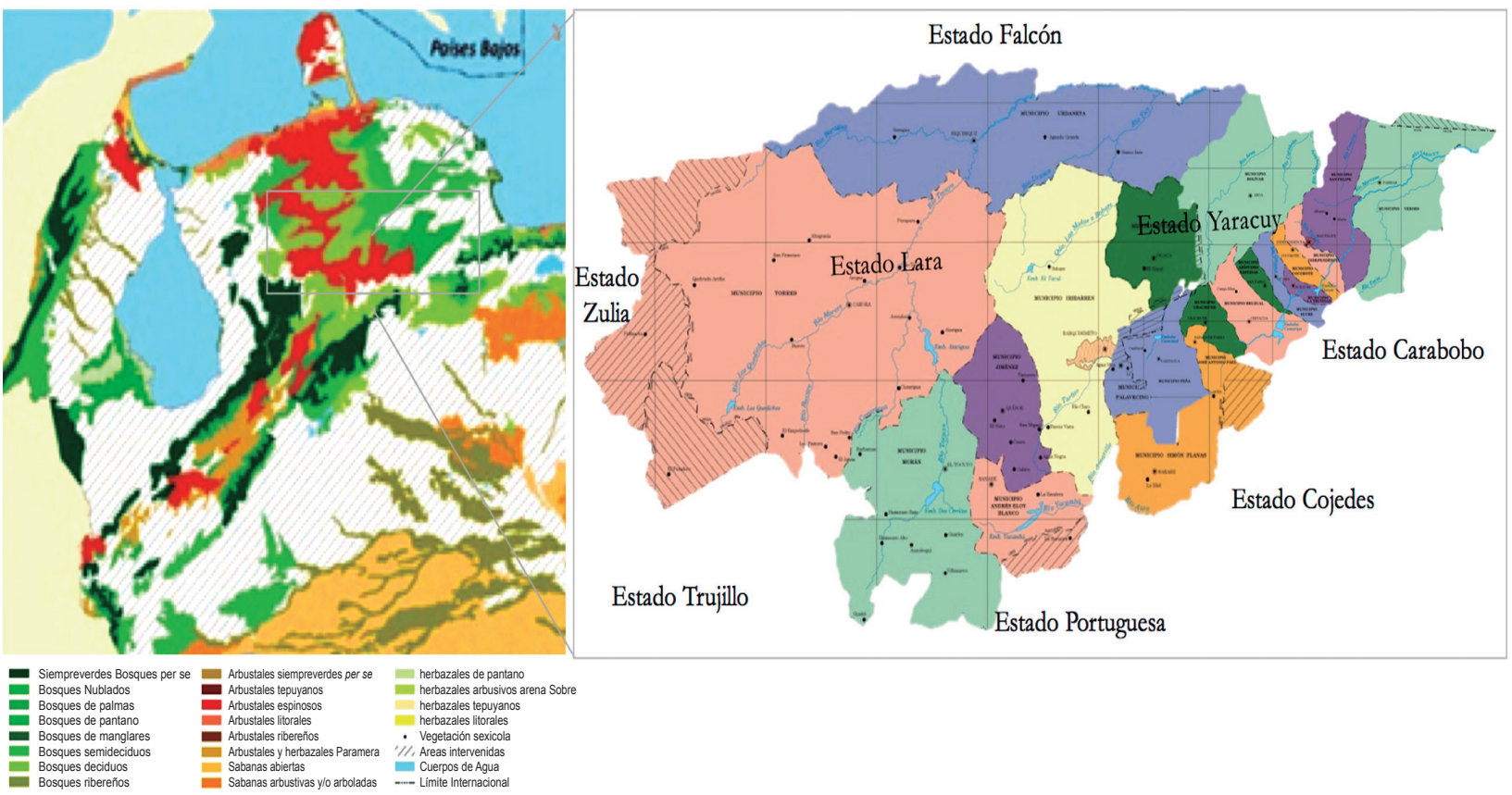

Figura 3. Posición geográfica de los estados de Lara y Yaracuy asociadas al muestreo de mieles florales venezolanas.

Tabla 1. Posicionamiento de las zonas apícolas relacionadas en el muestreo de mieles

\begin{tabular}{|c|c|c|c|c|c|c|}
\hline \multirow[t]{2}{*}{ Estado } & \multirow{2}{*}{ Localidad } & \multirow{2}{*}{$\mathrm{LN}$} & \multirow{2}{*}{ LO } & Cota & Precipitación & S. térmica \\
\hline & & & & $\mathrm{msnm}$ & $\mathrm{mm} / \mathrm{año}$ & ${ }^{\circ} \mathrm{C}$ \\
\hline \multirow{7}{*}{ Lara } & Crespo & $10^{\circ} 19^{\prime} 10,50^{\prime \prime}$ & $69^{\circ} 08^{\prime} 40,95^{\prime \prime}$ & 1220 & $500-800$ & 23,5 \\
\hline & Simón Planas & $09^{\circ} 52^{\prime} 25,7 ”$ & $69^{\circ} 02 ’ 25,9^{\prime \prime}$ & 283 & 1600 & 24,0 \\
\hline & Palavecino & $09^{\circ} 47^{\prime} 14,84^{\prime \prime}$ & $69^{\circ} 01^{\prime} 30,83 ”$ & 1000 & $400-500$ & 24,5 \\
\hline & Jiménez & $09^{\circ} 58^{\prime} 54,53^{\prime \prime}$ & $69^{\circ} 31^{\prime} 35,06^{\prime \prime}$ & 500 & $400-500$ & 22,0 \\
\hline & Morán & $09^{\circ} 46^{\prime} 07,73^{\prime \prime}$ & $69^{\circ} 48^{\prime} 43,52^{\prime \prime}$ & 620 & 400 & 22,0 \\
\hline & A. E. Blanco & $09^{\circ} 45^{\prime} 22,20^{\prime \prime}$ & $69^{\circ} 31^{\prime} 15,74^{\prime \prime}$ & 300 & 1680 & 21,0 \\
\hline & Iribarren & $10^{\circ} 22^{\prime} 46,60^{\prime \prime}$ & $69^{\circ} 22^{\prime} 22,42^{\prime \prime}$ & 765 & 800 & 24,5 \\
\hline \multirow[t]{3}{*}{ Yaracuy } & J. A. Páez & $10^{\circ} 06^{\prime} 54,21^{\prime \prime}$ & $68^{\circ} 57^{\prime} 03,52^{\prime \prime}$ & 340 & 1370 & 27,0 \\
\hline & Peña & $10^{\circ} 08^{\prime} 00,54^{\prime \prime}$ & $69^{\circ} 02^{\prime} 16,53 ”$ & 675 & 1900 & 26,0 \\
\hline & Urachiche & $10^{\circ} 09^{\prime} 33,22^{\prime \prime}$ & $69^{\circ} 00^{\prime} 31,45^{\prime \prime}$ & 477 & $1800-1900$ & 25,0 \\
\hline
\end{tabular}

9 localidades de los estados de Lara (13 muestras) y Yaracuy (17), en Crespo, Simón Plana, Palavecino, Jiménez, Moran, Andrés Eloy Blancos, Irribarren al oeste de Barquisimeto, Peña y Urachiche, (Tabla 1) siguiendo criterios de aleatoriedad simple y con la participación de personal adscrito a la Estación Apícola de la Universidad Lisandro Alvarado y evaluadas en la Universidad del Tolima (Ibagué, Colombia). Las muestras se acondicionaron usando recipientes estériles y herméticos de vidrio de 200 $\mathrm{mL}$ que se mantuvieron de manera individual en refrigerador a $5^{\circ} \mathrm{C}$, hasta el momento del análisis.

\subsection{Caracterizaciones}

En la valoración de la miel se aplicaron los métodos armonizados de análisis de mieles para los parámetros de humedad ( $X_{w}$, g./ 100 g.); azúcares totales $\left(X_{T}\right)$ y reductores $\left(X_{R}\right.$ fructosa, glucosa y sacarosa) g. $/ 100$ g., acidez meq. / kg., total $\left(A_{T}\right)$ lactónica $\left(A_{L}\right)$ y libre $\left(A_{L}\right)$; Glucosa (g. $/ 100$ g.) y la relación glucosa/Humedad $(\mathrm{G} / \mathrm{H})$; Color (CIELab y Pfund); actividad de agua $\left(a_{w}\right)$ Conductividad eléctrica (CE, mS. / cm.); Índice de diastasas (ID) y estudio palinológico de verificación del origen floral. Las muestras evaluadas se clasificaron según su apariencia, como mieles cristalizadas y líquidas. 


\subsection{Color y parámetros de cromaticidad}

Los parámetros de cromaticidad fueron evaluados siguiendo la técnica de análisis triestímulo para 30 longitudes de onda en el rango 400-700 nm, evaluando la transmitancia de mieles líquidas, filtradas y homogenizadas a través de un tamiz de $200 \mathrm{~mm}$. de paso y centrifugadas a $4000 \mathrm{rpm}$. por 10 min. Las determinaciones se realizaron en celdas de cuarzo de $10 \mathrm{~mm}$. de paso. Los parámetros de trabajo han sido luminancia (L), cromaticidad rojo/verde $\left(a^{*}\right)$, amarillo azul $\left(b^{*}\right)$ y croma (C). El iluminante de referencia ha sido C (Xn 98,4; Yn 100 y Zn 118,1), los parámetros de $\mathrm{L}, a^{*} b^{*}$ y $\mathrm{C}$ se determinaron de las relaciones que se describen en las siguientes ecuaciones:

$$
\begin{array}{cc}
L=\left[116\left[\frac{Y}{Y_{n}}\right]^{1 / 3}-16\right] & a *=500\left[\frac{X}{X_{n}}\right]^{1 / 3}-\left[\frac{Y}{Y_{n}}\right]^{1 / 3} \\
C=\left[\mathrm{a}^{* 2}+\mathrm{b}^{* 2}\right]^{1 / 2} & b *=200\left[\frac{Y}{Y_{n}}\right]^{1 / 3}-\left[\frac{Z}{Z_{n}}\right]^{1 / 3}
\end{array}
$$

La pureza de color se determinó interpolando los valores derivados de las relaciones triestímulo $Y_{n}, X_{n}$ y $Z_{n}$ en el locus de color, derivados para $\mathrm{x}$, $\mathrm{y}$, considerando los valores normalizados de $\mathrm{X}, \mathrm{Y} \mathrm{y}$ $\mathrm{Z}$ estimados en las determinaciones de transmitancia [49]. Adicionalmente el color natural de las mieles, se evaluó haciendo uso del fotómetro Hanna $^{T M}$ HI96785 para determinaciones en la escala Pfund, usando glicerina como patrón de calibración.

\subsection{Densidad óptica}

Se evaluaron disoluciones de miel, $(5,00 \mathrm{~g} \pm$ $1 \mathrm{mg}$ ), en $20 \mathrm{~mL}$. de agua destilada en la unidad espectrofotométrica UV-Visible Thermo Scientific ${ }^{\mathrm{TM}}$ Genesys $10 \mathrm{~S}$ a $420 \mathrm{~nm}$., en cubetas de cuarzo de 10 $\mathrm{mm}$. de paso espectral, a fin de relacionar valores de referencia respecto de las mediciones de color Pfund.

\subsection{Valoraciones sensoriales}

Los atributos sensoriales de las mieles fueron evaluados por etapas, en la primera sesión se presentaron los fundamentos del análisis sensorial y relacionando aromas relativos a la calidad de las mieles, conforme a los criterios descritos en la literatura [18]. Un total de 7 panelistas, sin revelar la identidad, describió de manera genérica y específica los estándares de referencia y reconociendo su identidad, los criterios de calidad y atributos de las mieles, entorno a la intensidad del aroma, color, cristalización, fluidez y sabor, haciendo uso de una escala hedónica de clasificación de 0 a 9 , con valores de 0 a 2 para atributos deficientes, 3 a 6 aceptables y 7 a 9 apropiados. Se aplicaron criterios del análisis cuantitativo descriptivo. En las perfilaciones se evaluaron aspectos globales (color, granulación, fluidez, aromas), olfativos (floral/afrutado, fresco, herbal, humo y madera) y gustativos (acidez, dulzor, fermentado, metalicidad y retrogusto), conforme a las directrices de CIE.

\subsection{Análisis multivariado}

En la evaluación de funciones discriminantes realizadas, se consideraron 7 variables predictoras que reflejan la calidad global de los tipos de mieles, $\left(\mathrm{L}, a^{*} / b^{*}, \mathrm{pH}\right.$, humedad $\left(X_{H}\right)$, conductividad eléctrica (CE) y color de la escala Pfund (mm.). Los resultados asociados a grupos de muestras, que generaron funciones de clasificación con valor-P inferiores a 0.05 fueron consideradas estadísticamente significativas. En el análisis de clasificación por conglomerados se usaron los parámetros $\left(a^{*} / b^{*}\right)$, $\mathrm{CE}$, humedad $\left(X_{H}\right), \mathrm{pH}$, longitud de onda dominante y la pureza de color; en el sistema Bayesiano de redes neuronales $\left(X_{A L}\right)$, azúcares totales $\left(X_{S}\right)$, Cenizas y $\mathrm{pH}$. En la evaluación estadística se usaron los paquetes Statgraphic centurion ${ }^{T M}$ XVI, V 16.1.032.

\subsection{Correlaciones canónicas y PLS}

Con la información generada de las propiedades fisicoquímicas y sensoriales obtenidas en el trabajo de caracterización, se realizó un estudio de las relaciones sensoriales y los parámetros fisicoquímicos, mediante técnicas de PLS, procurando una metodología que permita cuantificar las relaciones de las caracterizaciones. Se usaron los paquetes estadísticos XLSTAT $T^{T M}$, Infostat ${ }^{T M}$, Minitab $16^{T M}$, Statistica 10.

\section{Resultados y Discusión}

\subsection{El sistema apícola}

La fracción geográfica de los estados de Lara y Yaracuy,seestructuraenlaunidadcorianadetransición de los Andes a la costa caribe venezolana. En esta unidad se observaron diversos tipos de vegetación, (xerófila, bosques deciduos y semideciduos), como también áreas de arbustos espinosos y cardenales, 
que son aprovechadas por grupos de apicultores con una reducida capacidad instalada. La condición más frecuente de estas comunidades vegetales es encontrar una mezcla entre espinares y cardonales. La composición de especies de cada unidad está influenciada por la disponibilidad de agua, los tipos de suelo y su salinidad. Los bosques deciduos son menos diversos que los bosques húmedos. La flora arbustiva distribuida está constituida por especies de las familias Mimosaceae, Caesalpinae, Caparidaceae de los géneros Prosopis, Acacia, Cercidium y Capparis. Se observaron cactáceas columnares emergentes, de los géneros Stenocereus, Subpilocereus y Pilosocereus. Igualmente se destacaron especies de los géneros Croton, Opuntia, Jatropha y Cnidoscolus y plantas herbáceas de los géneros Lantana, Digitaria, Evolvulus, Sida, Sporobolus y cactáceas de los géneros Opuntia, Mammillaria y Melocactus, relacionados en los trabajos del grupo de trabajo de Barrios [52].

\subsection{Parámetros fisicoquímicos}

Los parámetros asociados a las muestras analizadas se recogen en la tabla 2. El contenido de cenizas osciló entre $0,275(\mathrm{~g} / 100 \mathrm{~g}$. miel) propio de mieles ámbar claro y 0,367 (g/100 g miel), en general las muestras del estado Lara, fueron parecidas a las beneficiadas en las localidades de Yaracuy, concordantes con otras mieles venezolanas analizadas [45] al igual que de Marruecos [54], Eslovenia [55] y diferentes a los observados por investigadores de la IHC [48], este parámetro fue usado para determinar el origen botánico de las mieles. En este estudio, la conductividad eléctrica de muestras al $20 \%$ en sólidos solubles presentaron tonalidad ámbar claro (LA) y Ámbar (A) con valores entre 0,692 a $0,777 \mathrm{mS} . \mathrm{cm}^{-1}$, coincidentes con las observaciones y patrones de mieles de España y Portugal [16]. Usualmente el color de las mieles con bajos contenidos de minerales, han sido relacionadas con tonos de miel claro y bajos niveles de conductividad, las muestras obscuras por su parte con una mayor fracción de materiales solubles [5658]. La figura 9, mostró valores concordantes con la información que se relaciona.
La fracción de azúcares de las mieles analizadas en el estudio, presentó un 13\% de las muestras con una fuerte tendencia a la cristalización natural, la relación $\mathrm{G} / \mathrm{H}$ en éste tipo de muestras fue de 1.762 (Sanare), similar en el caso de las de (Cubiro) y con 1.82 las de Guárico y La Lucia, del estado Lara. En Yaracuy se presentaron mieles con tendencia a la cristalización, con 1,792 en la relación $(\mathrm{G} / \mathrm{H})$, en muestras de Guaremal y Cambural, con valores de 1,862 . El contenido de humedad $(X w, \mathrm{~g} / 100 \mathrm{~g})$ y la fracción de azúcares totales $(X s, \mathrm{~g} / 100 \mathrm{~g})$ entre grupos de mieles no presentó diferencias significativas, pero si respecto de los grupos por zonas geográfica $(\mathrm{Pv}<$ $0,05)$. Éste mismo comportamiento prevaleció sobre los parámetros de actividad de agua $\left(a_{w}\right)$ y densidad (g. $m L^{-1}$ ).

Las evaluaciones relativas al color en términos de longitud de onda dominante presentaron diferencias importantes entre grupos de muestras $(\mathrm{Pv}<0,05)$ y con ello la pureza del color los parámetros de cromaticidad $\left(a^{*} / b^{*}\right)$, como en los valores finales de las determinaciones del color en la escala Pfund. El análisis polínico en particular no reveló composición monofloral, las muestras mostraton polen aislado y acompañante que indica que el origen botánico fue multifloral (datos no mostrados), coincidiendo con el reporte para distintos tipos de mieles venezolanas [45]. Las mediciones de color en la escala Pfund, reveló tres tipos de muestras coincidentes con los tipos ámbar (A. 85-114 mm), ámbar claro (LA. 50-85 mm) y ámbar obscura (DA. >114 mm). Los dos primeros tipos fueron comunes a los estados de Lara y Yaracuy, posiblemente debido a las mismas condiciones de entorno y oferta floral. Los parámetros definidos en este estudio en relación a los tipos de mieles se ilustran en la Tabla 2. La muestras exhibieron longitudes de onda entre 573 nm. (Sarare) a $605 \mathrm{~nm}$. (Los Higuitos). Los valores de cromaticidad observados oscilaron entre 575 y $586 \mathrm{~nm}$. en Yaracuy. En las figuras 4 y 5, se muestra la distribución cromática en el espacio $\left(a^{*} / b^{*}\right)$, con las secuencias de longitudes de onda dominantes y la distribución en el locus de color x-y. 
Tabla 2. Valores medios asociados a los parámetros fisicoquímicos de mieles venezolanas por estado y por tonalidades de color [14].

\begin{tabular}{lccccc}
\hline \multicolumn{1}{c}{ Parámetros } & Lara $(\mathrm{ds})$ & Yaracuy $(\mathrm{ds})$ & $\mathrm{A}(\mathrm{ds})$ & LA $(\mathrm{ds})$ & DA $(\mathrm{ds})$ \\
\hline$a_{w}$ & $0,573(0,007)$ & $0,540(0,010)$ & $0,559(0,007)$ & $0,545(0,008)$ & $0,565(0,017)$ \\
$\mathrm{pH}$ & $3,544(0,036)$ & $3,448(0,047)$ & $3,466(0,035)$ & $3,424(0,040)$ & $3,598(0,082)$ \\
$\mathrm{X}_{\mathrm{AL}}$ & $34,6(1,71)$ & $34,1(2,27)$ & $34,1(1,70)$ & $29,8(1,88)$ & $39,3(3,95)$ \\
$\mathrm{X} w$ & $20,9(0,30)$ & $19,8(0,41)$ & $20,3(0,30)$ & $20,0(0,34)$ & $20,7(0,30)$ \\
$\mathrm{Xs}$ & $79,1(0,30)$ & $80,2(0,41)$ & $79,7(0,30)$ & $79,9(0,34)$ & $79,3(0,71)$ \\
Glucosa $(\% \mathrm{p} / \mathrm{p})$ & $33,5(0,86)$ & $31,6(1,14)$ & $33,2(0,85)$ & $32,8(0,94)$ & $31,6(2,00)$ \\
$\mathrm{CE}\left(\mathrm{mS} . \mathrm{cm}^{-1}\right)$ & $0,674(0,087)$ & $0,714(0,110)$ & $0,777(0,086)$ & $0,613(0,096)$ & $0,692(0,201)$ \\
Xc & $0,311(0,050)$ & $0,330(0,060)$ & $0,367(0,050)$ & $0,275(0,050)$ & $0,314(0,106)$ \\
Densidad $\left(\mathrm{g} . \mathrm{mL}^{-1}\right)$ & $1,4078(0,002)$ & $1,4157(0,002)$ & $1,4121(0,002)$ & $1,4141(0,002)$ & $1,4092(0,004)$ \\
Color $($ Pfund) & $98,8(2,30)$ & $100,5(3,03)$ & $99,1(2,25)$ & $71,1(2,51)$ & $128,1(5,30)$ \\
L & $47,4(1,40)$ & $46,4(1,82)$ & $47,3(1,40)$ & $64,1(1,50)^{b}$ & $29,3(3,20)$ \\
$a^{*}$ & $19,1(1,90)$ & $17,2(2,60)$ & $15,9(1,90)$ & $1,41(1,12)^{b}$ & $37,2(4,45)$ \\
$b^{*}$ & $54,7(1,10)$ & $53,7(1,43)$ & $54,7(1,06)$ & $67,8(1,20)^{b}$ & $40,1(2,50)$ \\
C & $61,1(1,16)$ & $59,7(1,53)$ & $57,4(1,14)$ & $68,1(1,27)$ & $55,5(2,70)$ \\
h & $68,3(1,60)$ & $69,9(2,12)$ & $73,8(1,60)$ & $85,2(1,80)$ & $48,3(3,70)$ \\
L. Onda D. & $588,1(0,94)$ & $587,6(1,25)$ & $584,4(0,93)$ & $577,4(1,03)$ & $601,7(2,17)$ \\
Pureza color & $81,1(0,62)$ & $81,9(0,82)$ & $80,0(0,61)$ & $79,2(0,70)$ & $85,4(1,42)$ \\
\hline
\end{tabular}

Acidez libre, $\left(\mathrm{X}_{\mathrm{AL}}\right)$. Humedad $(\mathrm{X} w)$. Azucares totales $(\mathrm{X} s)$. Cenizas $(\mathrm{Xc})$. Cromaticidad $\left(a^{*} / b^{*}\right)$. Croma $(\mathrm{C})$. Angulo de tono (h). Luminancia (L).

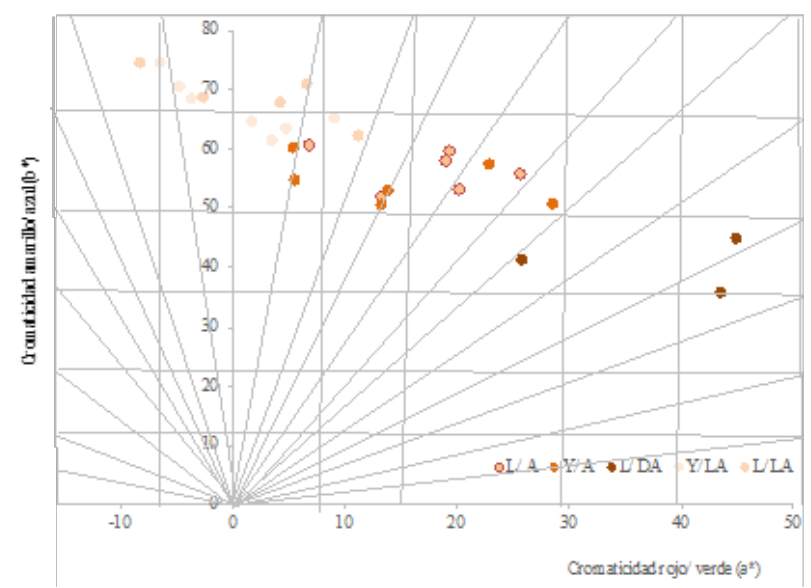

Figura 4. Distribución cromática $a^{*} / b^{*}$ de los tipos de mieles venezolanas de los estados de Lara y Yaracuy.

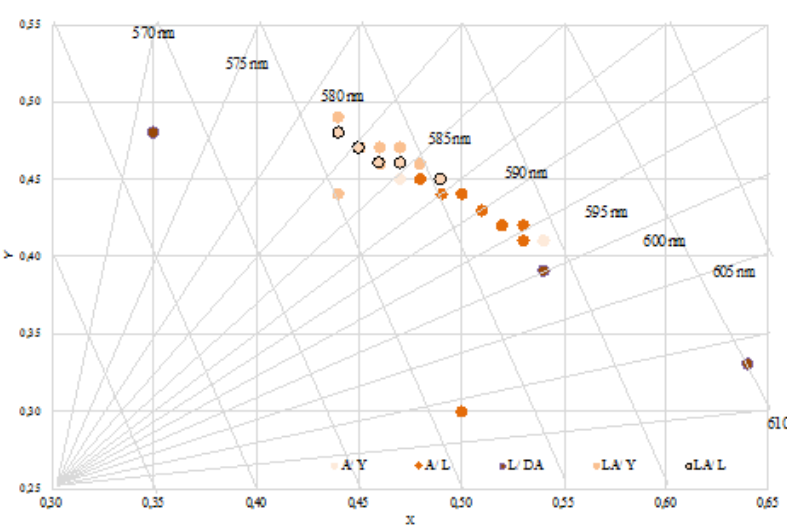

Figura 5. Tipos de mieles venezolanas de los estados de Lara y Yaracuy y sus longitudes de onda en el locus de color $\mathrm{x}-\mathrm{y}$.

\subsection{Valoraciones sensoriales}

Los tipos de mieles predominantes en las zonas de Lara y Yaracuy, correspondieron a los tipos A y LA, su intensidad en cada tipo es marcada (Figura 6). Las mieles LA, presentaron aromas finos y tenues con notas florales o afrutadas de perfil débilmente ácido, tenor fragante y persistente, de notas dulces y marcado retrogusto la mayoría de las muestras no tuvieron granulación ni sedimentos, el retrogusto al dulce y la granulación fina y homogénea predominaron en las muestras cristalizadas. El grupo A y DA, presentó matices brillantes, aroma floral pronunciado y retrogusto persistente. En todos los casos los tenores metálicos se expresaron débilmente o estuvieron ausentes, se percibieron notas de humos que no fueron dominantes. Los perfiles sensoriales de la escala hedónica (Figura 7), para fluidez no presentaron diferencias significativas entre los grupos según su tonalidad, si por razones obvias en el color y el sabor.

\subsection{Conglomerados y redes neuronales}

El análisis de conglomerados reveló tres grupos definidos de mieles y su entorno geográfico (Figura, 8 ), en el primer grupo aparecieron mieles del tipo ambar claro, (LA: 56,5-84,4 mm), con $40 \%$ del total muestreado, respecto de la pureza de color se evidenció que las mieles fueron de tonalidades 


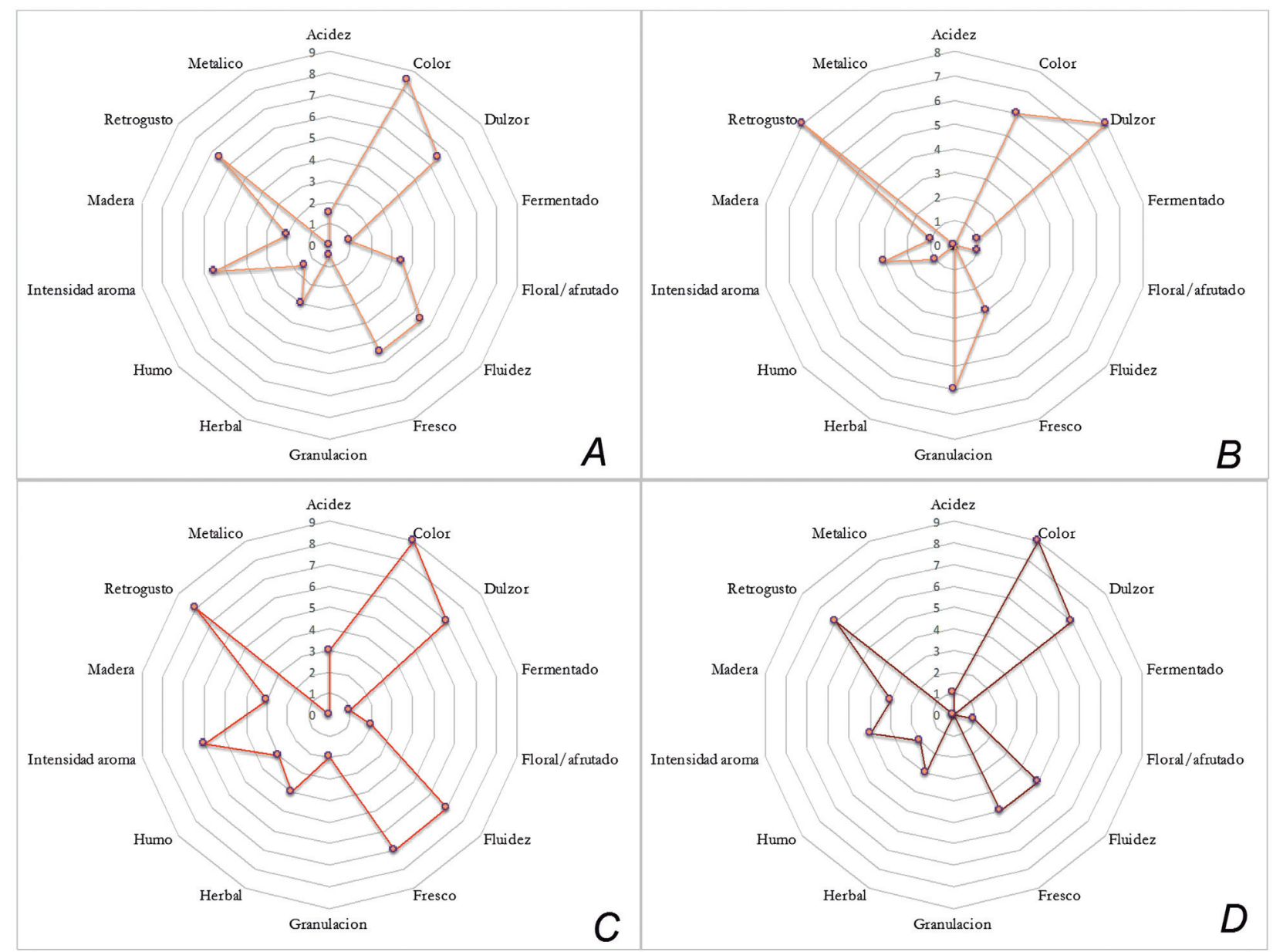

Figura 6. Perfiles sensoriales tipo de las mieles venezolanas de Lara y Yaracuy. A. Ambar claro líquida, B. Ambar claro cristalizada, $C$. Ambar, D. Ambar obscura.

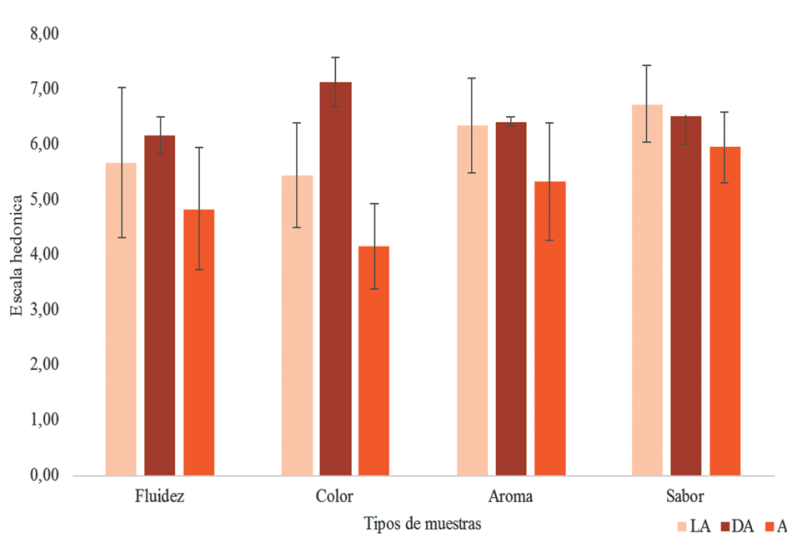

Figura 7. Dendograma de clasificación de los tipos de mieles venezolanas de los estados de Lara y Yaracuy y sus entornos

amarillo y amarillo naranja, las longitudes de onda dominante oscilaron entre el rango $573-580 \mathrm{~nm}$., el pH fue propio de mieles multiflorales que se relacionaron con valores de Acidez libre, con alguna tendencia a la cristalización en virtud a la relación glucosa/agua. El segundo grupo se relacionó con muestras tipo ámbar (A: 86,4-106,9 mm), representaron el $50 \%$ de las mieles analizadas, la pureza de color reveló que fueron mieles de tonalidad amarillo naranja, la conductividad eléctrica de 0,41 a $1,78 \mathrm{mS} . \mathrm{cm}^{-1}$, para humedades que oscilaron entre 18,1 a 22,3 $\mathrm{g} / 100 \mathrm{~g}$, la actividad de agua $\left(a_{w}: 0,498\right.$ a 0,605$)$, principalmente fueron mieles líquidas y fluidas de baja tendencia a la cristalización, $\mathrm{pH}(3,33$ a 3,70$)$ y acidez de 26.7 a 46.9 meq. $\mathrm{Kg}^{-1}$. Finalmente el grupo ámbar obscuro (DA: 122 y $137 \mathrm{~mm}$ ), fue un grupo de muestras minoritario representado solo con $10 \%$ del total muestreado, la pureza de color correspondió a tipos de mieles del naranja-rojizo de longitudes de onda dominante (LD: 595 a $605 \mathrm{~nm}$ ), conductividad eléctrica (CE: 0,32 a $\left.0,87 \mathrm{mS} . \mathrm{cm}^{-1}\right)$ y cenizas $(0,12$ a $0,41 \mathrm{~g} / 100 \mathrm{~g}$ ), pH desde 3,53 a 3,78 concordante con la acidez de 31.4 a 53.6 meq. $\mathrm{Kg}^{-1}$, la humedad de 21,1 a 22,2 con $a_{w}$ de 0,543 a 0,605 y la relación de glucosa/humedad de 1.376 a 1.762 . Los atributos sensoriales entre los grupos de muestras presentaron diferencias significativas, en virtud a las propiedades intrínsecas de las mieles evaluadas. 


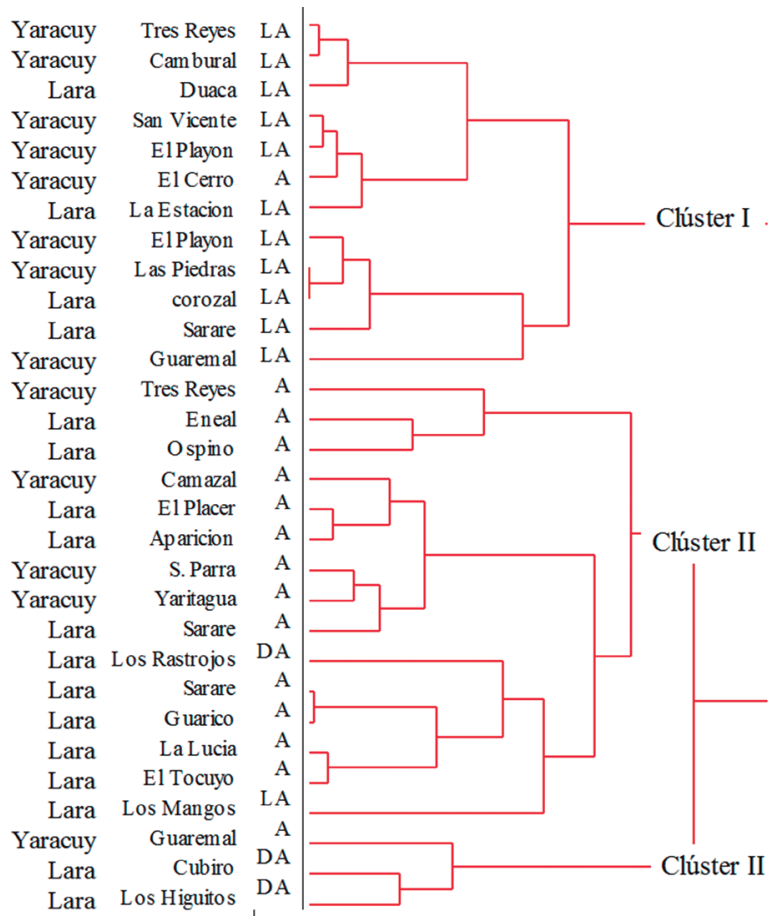

Figura 8. Dendograma de clasificación de los tipos de mieles venezolanas de los estados de Lara y Yaracuy y sus entornos

El diagrama de la figura 9, relaciona la red neuronal para los tipos de mieles conforme a la clasificación del sistema USDA [14] y el diagrama de contorno de los casos de trabajo conducentes a conjuntos de entrenamiento y clasificación final de los grupos dominantes, ámbar y ámbar claro. Los 30 casos del conjunto de entrenamiento, fueron clasificados por la red neuronal generada. El origen geográfico y el aporte de flujos de néctar en periodos distintos es sugerido.

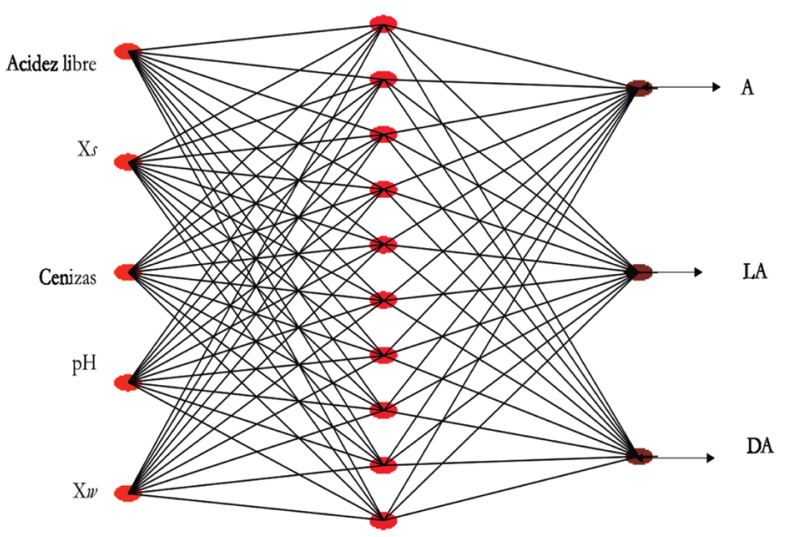

Figura 9. Red neuronal de clasificación de los tipos de mieles venezolanas de Lara y Yaracuy.

\subsection{Análisis discriminante}

Los parámetros de referencia seleccionados para el estudio de clasificación de las muestras marcan diferencias entre mieles del tipo Ámbar, Ámbar obscuro y Ámbar claro. Las funciones de clasificación para los tres tipos de mieles observadas se ajustó a una relación lineal de la forma: $\quad \beta_{0}+\beta_{1} a^{*}+\beta_{2} b^{*}+\beta_{3} \mathrm{CE}+\beta_{4} X w+\beta_{5} p H+\beta_{6}$ Pfund $+\beta_{7} L$. Los promedios y desviaciones estándar de cada tipo de miel estuvieron en función de la guía de clasificación del color (Tabla 3). Los resultados de la categorización mediante análisis discriminante se recogen en la tabla 4. Nótese las diferencias en la cromaticidad rojo/verde $\left(a^{*}\right)$, desde 1,26 \pm 0.63 a $38,0 \pm 1.07$. Éste parámetro, como también L y $\left(b^{*}\right)$ contribuyeron a la diferenciación de las mieles (Figura 4).

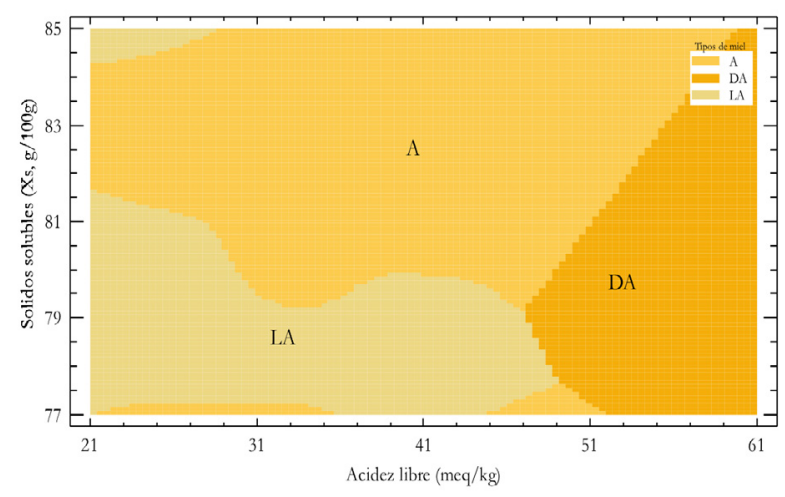

Figura 10. Representación de la clasificación de los tipos de mieles por tonalidad de color Pfund.

Tabla 3. Coeficientes de las funciones discriminantes para la clasificación de tipos de mieles venezolanas de Lara y Yaracuy.

\begin{tabular}{|l|c|c|c|}
\hline Coeficientes & Ámbar & Ámbar obscuro & Ámbar claro \\
\hline$\beta_{0}$ & $-3,03032 \mathrm{E} 9$ & $-3,03036 \mathrm{E} 9$ & $-3,03034 \mathrm{E} 9$ \\
$a^{*}$ & $-3727,0$ & $-3726,07$ & $-3727,22$ \\
$b^{*}$ & 8326,49 & 8321,82 & 8327,44 \\
\hline $\mathrm{CE}$ & 574,553 & 574,783 & 574,111 \\
\hline $\mathrm{X} w$ & 22706,0 & 22705,4 & 22706,1 \\
\hline $\mathrm{pH}$ & -251316 & -251308 & -251320 \\
\hline Color (Pfund) & $3,4157 \mathrm{E} 7$ & $3,41572 \mathrm{E} 7$ & $3,41571 \mathrm{E} 7$ \\
\hline $\mathrm{L}$ & $5,66376 \mathrm{E} 7$ & $5,66379 \mathrm{E} 7$ & $5,66377 \mathrm{E} 7$ \\
\hline
\end{tabular}

Tabla 4. Valores medios y desviaciones estándar de los grupos de mieles de los estados de Lara y Yaracuy.

\begin{tabular}{|l|c|c|c|c|}
\hline Tipo & A(ds) & DA (ds) & LA(ds) & Total (ds) \\
\hline$\%$ & 50 & 10 & 40 & 100 \\
\hline$n$ & 15 & 3 & 12 & 30 \\
\hline$a^{*}$ & $16,1(7,24)$ & $38,0(1,07)$ & $1,26(0,63)$ & $12,3(1,30)$ \\
\hline$b^{*}$ & $54,8(3,60)$ & $40,7(4,55)$ & $67,8(4,44)$ & $58,6(9,50)$ \\
\hline $\mathrm{CE}\left(\mathrm{mS} . \mathrm{cm}^{-1}\right)$ & $0,773(0,130)$ & $0,672(0,306)$ & $0,616(0,180)$ & $0,700(0,321)$ \\
\hline $\mathrm{X} w$ & $20,4(4,04)$ & $21,3(1,10)$ & $19,9(1,28)$ & $20,3(1,30)$ \\
\hline $\mathrm{pH}$ & $3,47(0,11)$ & $3,64(0,12)$ & $3,41(0,16)$ & $3,47(0,15)$ \\
\hline Pfund $(\mathrm{mm})$ & $98,8(7,70)$ & $128(7,70)$ & $71,3(8,36)$ & $90,7(20,1)$ \\
\hline $\mathrm{L}$ & $47,3(4,64)$ & $29,8(8,36)$ & $64,0(5,72)$ & $52,3(12,0)$ \\
\hline
\end{tabular}

$n$ : Número de datos. $a^{*}$ : Cromaticidad rojo/verde, $b^{*}$ : Amarillo/azul. CE: Conductividad eléctrica.

$\mathrm{Xw}$ : humedad (g/100). pH: Potencial de hidrógeno.

Color (Pfund). L: Luminancia. 


\subsection{Correlaciones canónicas y PLS-PATH}

El modelo generado se asocia a tablas múltiples (Tabla 5) y dos submodelos, uno de ellos estructural o interno relacionado con parámetros fisicoquímicos de los tipos de mieles y en particular los concernientes a luminancia (L), cromaticidad $\left(a^{*} / b^{*}\right)$, pureza del color y los criterios hedónicos de los jueces asociados al análisis cuantitativo descriptivo (QDA) y el de medición o externo se ilustra en la Figura 12. En la secuencia de clasificación mediante análisis de componentes principales, revelaron los aportes de las variables de estudio al origen de las muestras en virtud a su origen geográfico (Figuras 11 y 12), como proyección factorial de los atributos representativos de las mieles. En el diagnóstico se cumplieron los supuestos del modelo de regresión en términos de normalidad e independencia de los residuales y homocedasticidad en las observaciones (Tabla 6). Las evaluaciones en términos de componentes principales $\left(t_{1}\right.$ y $\left.t_{2}\right)$, mostraron una dimensionalidad diferente para cada uno de los grupos.

Tabla 5. Parámetros fisicoquímicos, sensoriales y hedónicos tipo de mieles venezolanas.

\begin{tabular}{|c|c|c|c|c|c|c|}
\hline \multirow{2}{*}{ Muestras } & VE05. & VE09... & VE12 & \multirow{2}{*}{\multicolumn{3}{|c|}{$\begin{array}{l}\text { VE10.. VE28.. VE29 } \\
\text { Estado Lara }\end{array}$}} \\
\hline & \multicolumn{3}{|c|}{ Estado Yaracuy } & & & \\
\hline \multicolumn{7}{|l|}{ Fisicoquímicos } \\
\hline $\mathrm{L}$ & 72,9 & 41,1 & 51,3 & 56,4 & 63,8 & 32,3 \\
\hline$a^{*}$ & $-6,50$ & 28,5 & 5,50 & 11,2 & 4,20 & 45,0 \\
\hline$b^{*}$ & 74,6 & 50,9 & 54,7 & 62,2 & 68,0 & 45,0 \\
\hline L. Onda D (nm) & 577 & 590 & 580 & 583 & 577 & 605 \\
\hline$X c$ & 0,23 & 0,38 & 0,23 & 0,34 & 0,23 & 0,64 \\
\hline $\mathrm{CE}\left(\mathrm{mS} . \mathrm{cm}^{-1}\right)$ & 0,528 & 0,816 & 0,525 & 0,737 & 0,533 & 1,290 \\
\hline Humedad & 20,0 & 20,7 & 20,0 & 20,7 & 21,5 & 21,5 \\
\hline$a_{w}$ & 0,50 & 0,60 & 0,60 & 0,60 & 0,60 & 0,60 \\
\hline $\mathrm{pH}$ & 3,40 & 3,60 & 3,50 & 3,50 & 3,90 & 3,50 \\
\hline $\mathrm{AT}$ (meq. $\mathrm{kg}^{-1}$ ) & 25,8 & 28,9 & 32,2 & 30,8 & 29,6 & 31,4 \\
\hline Glucosa/Agua & 1,60 & 1,60 & 1,40 & 1,60 & 1,60 & 1,40 \\
\hline \multicolumn{7}{|l|}{ Sensoriales } \\
\hline Fluidez & 5,90 & 4,40 & 8,30 & 5,30 & 4,40 & 6,50 \\
\hline Color & 3,60 & 5,30 & 5,10 & 3,90 & 6,00 & 7,70 \\
\hline Aroma & 4,90 & 5,40 & 7,30 & 4,40 & 6,60 & 6,50 \\
\hline Cristal & 0 & 0 & 4,00 & 1,30 & 0 & 0 \\
\hline Sabor & 6,10 & 7,60 & 6,70 & 6,30 & 7,10 & 6,20 \\
\hline \multicolumn{7}{|l|}{ Hedónicos } \\
\hline $\mathrm{J} 1$ & 7 & 8 & 6 & 3 & 8 & 9 \\
\hline & . & $\cdots$ & $\cdots$ & .. & $\cdots$ & .. \\
\hline J7 & 9 & 8 & 7 & 5 & 9 & 5 \\
\hline
\end{tabular}

En el trabajo el análisis de componentes principales $(\mathrm{CP})$ para los parámetros fisicoquímicos y en particular la luminancia $\mathrm{L}$ y cromaticidad $b^{*}$, mostró una asociación que representan el mayor aporte a la clasificación en el espacio factorial; así mismo, los atributos de cristalización y fluidez asociados a la segunda componente. Este mismo comportamiento, se evidenció en el plano de propiedades fisicoquímicas y jueces, Figura 11. Los parámetros fisicoquímicos y particularmente los correspondientes a la luminancia, cromaticidad $\left(a^{*} / b^{*}\right)$ y la pureza del color, contribuyeron a su clasificación, además los atributos intrínsecos y sensoriales, se correlacionan con los criterios de los jueces que participaron en el estudio de muestras mediante análisis cuantitativo descriptivo (QDA), Figura 13.

Tabla 6. Valores propios asociados al análisis de componentes principales

\begin{tabular}{ccc|cc|cc}
\hline \multirow{2}{*}{ Ejes } & \multicolumn{2}{c|}{ Fisicoquímicos } & \multicolumn{2}{c|}{ Sensoriales } & \multicolumn{2}{c}{ Jueces } \\
& V.propio & \%Inercia & V.propio & \%Inercia & V.propio & \%Inercia \\
\hline 1 & 4,19 & 42,0 & 2,10 & 34,0 & 3,00 & 43,0 \\
2 & 1,59 & 16,0 & 1,78 & 30,0 & 1,28 & 18,0 \\
3 & 1,17 & 12,0 & 0,69 & 12,0 & 0,98 & 14,0 \\
4 & 1,10 & 10,0 & 0,65 & 11,0 & 0,74 & 11,0 \\
\hline
\end{tabular}

El análisis de las dos primeras componentes principales, permitió clasificar el origen de las muestras en virtud a su origen geográfico. En el diagnóstico se cumplen los supuestos del modelo de regresión en términos de normalidad e independencia de los residuales y homocedasticidad en las observaciones.

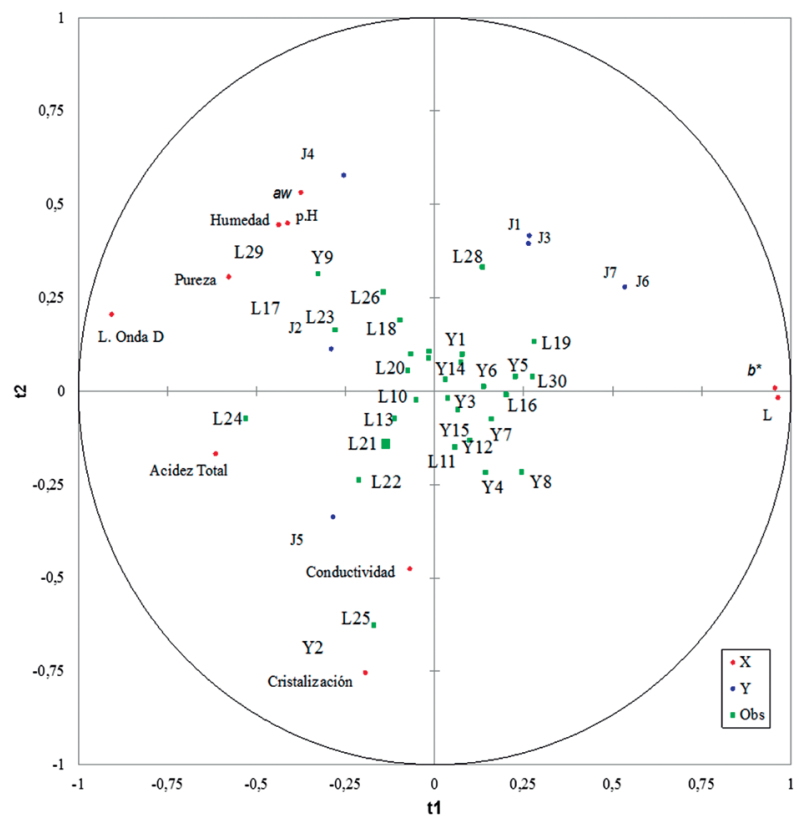

Figura 11. Proyección factorial de los atributos sensoriales, parámetros fisicoquímicos y panelistas en la clasificación de las mieles venezolanas de Lara y Yaracuy. 


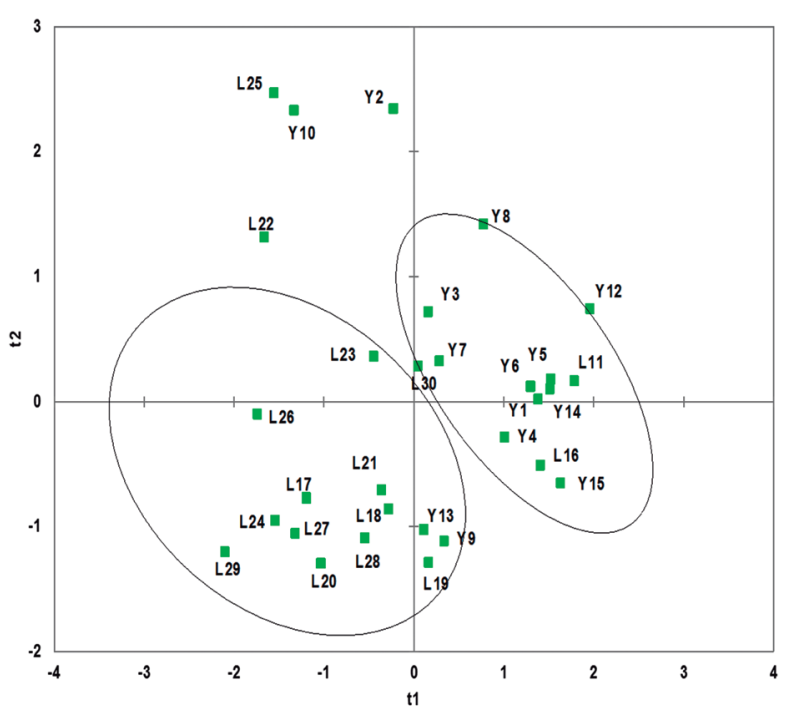

Figura 12. Distribución factorial de las muestras de miel de las zonas de Lara y Yaracuy.

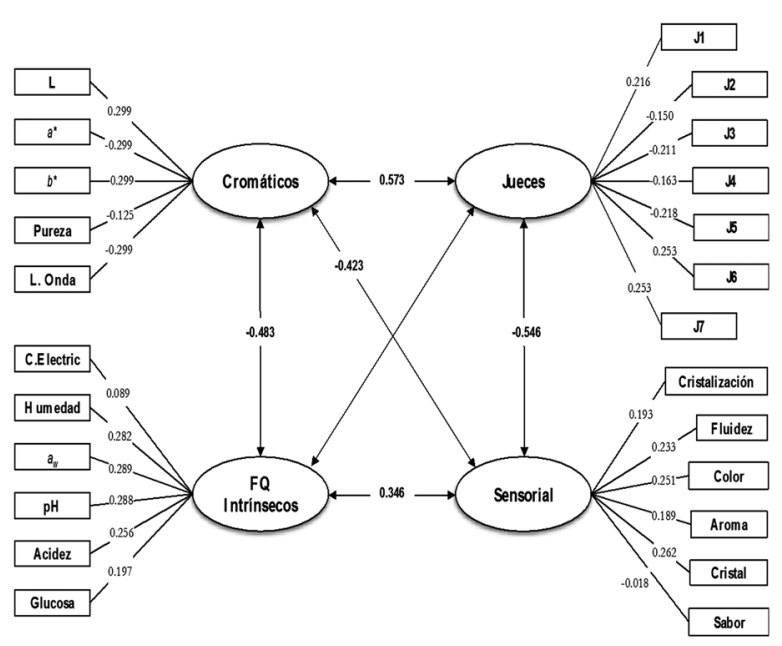

Figura 13. Relaciones PLS-PATH en la clasificación de mieles de los Estado Lara y Yaracuy a partir del análisis factorial múltiple.

\section{Conclusiones}

El trabajo realizado permitió evaluar y estudiar las propiedades fisicoquímicas de mieles, cuyos resultados lograron formular y generar criterios de clasificación, haciendo uso de técnicas de análisis multivariado en la construcción de un espacio que interrelaciona las propiedades intrínsecas de las mieles venezolanas de los estados de Lara y Yaracuy y sus atributos sensoriales. Los parámetros intrínsecos de acidez libre, actividad de agua, densidad, conductividad eléctrica, humedad, potencial de hidrógeno, color y cromaticidad, contribuyeron a los procesos de clasificación y diferenciación de las muestras. Estos parámetros permitieron identificar tres grupos de mieles con propiedades y atributos diferenciables. Los criterios sensoriales y análisis hedónico posibilitaron la comprensión y clasificación de grupos de muestras con atributos similares de manera independientemente del origen geográfico, condición que se ratifica a través del análisis de discriminante, por conglomerados y redes neuronales. El modelamiento de clasificación mediante técnicas de PLS-PATH, permitió cuantificar el efecto que tienen las características y atributos de las mieles evaluadas en función del origen geográfico. El estudio se consolida como modelo de evaluación y clasificación de mieles, complementa otros trabajos reportados en la literatura sobre calidad de mieles venezolanas y da apertura a nuevas formas de evaluación y de clasificación mediante técnicas de análisis robusto.

\section{Agradecimientos}

Los autores expresan su gratitud a los campesinos y apicultores de las localidades de Crespo, Palavecino, Moran, A.E. Blanco, J.A. Páez, Peña y Urariche en los estados de Lara y Yaracuy, por las condiciones facilitadas durante las operaciones de muestreo. A los profesores Judith Principal, Zaida Graterol, Carlos Barrios y al personal técnico de la estación apícola de la Universidad Centro Occidental Lisandro Alvarado, por la consolidación del muestreo y las condiciones de traslado de las muestras a la Universidad del Tolima en Colombia. Al equipo de trabajo del Grupo de Investigaciones Mellitopalinológicas y Propiedades Fisicoquímicas de Alimentos de la Universidad del Tolima, por el trabajo adelantado en el proceso de caracterización. Finalmente expresamos toda nuestra gratitud a los evaluadores del artículo y los colaboradores permanentes de la revista Ciencia en Desarrollo, por las observaciones, sugerencias y recomendaciones al documento final durante el proceso de arbitraje.

\section{Referencias}

[1] G. Nayik, B. Dar, V. Nanda, "Physicochemical, rheological and sugar profile of different unifloral honeys from Kashmir valley of India", Arabian Journal of Chemistry, In Press, 2015.

[2] V. Da Silva, L. Andrade, N. Leite, R. De Almeida, J. Vilela. "Rheological and Thermal Properties of Selected Brazilian Honeys from Various Floral Origins", Journal of Texture Studies, vol. 47(3): 208-219. 2016.

[3] J.M Alvarez-Suarez, F. Giampieri, M. Battino, M. Honey as a source of dietary antioxidants: Structures, bioavailability and evidence of 
protective effects against human chronic diseases. Curr. Med. Chem. 20, 621-638. 2013.

[4] V. M. Da Silva, L. Andrade De Carvalho., N.L. De Oliveira, R. De Almeida -Torres Filho, R., J. Vilela De-Resedente. "Rheological and thermal properties of selected brazilian honeys from various floral origins"Journal of Texture Studies 47, 208-219. 2016.

[5] R. Ramírez, G. Montenegro. "Certificación del origen botánico de miel y polen corbicular pertenecientes a la comuna de Litueche, VI región de Chile", Ciencia e Investigación Agraria, vol. 31, pp. 197-211, 2004.

[6] O. Persano, L. Piro. "Main European unifloral honeys descriptive sheets", Apidogie, vol. 35, pp. S38-S81, 2004.

[7] M. Urueña, E. Arrieta, E. Umaña, L. Zamora, M. Arias, "Evaluación de la posible adulteración de mieles de abeja comerciales de origen costarricence al compararlas con mieles artesanales provenientes de apiarios específicos", Archivos Latinoamericanos de Nutrición, vol. 57(1), pp. 63-68, 2004.

[8] S. Bogdanov, C. Lullmann, "Honey Quality and International Regulatory Standards, Review by the International Honey Commission, Bee Department, Federal Dairy Research Institute, Suiza", 1998.

[9] Y. Moguel, C. Echazarreta, R. Mora, "Calidad fisicoquímica de la miel de abeja Apis mellifera producida en el estado de Yucatán durante diferentes etapas del proceso de producción y tipos de floración", Téc Peru Méx, vol. 43(3), pp. 323-334, 2005.

[10] G. Salamanca, "Estudio y clasificación de mieles colombianas mediante redes neuronales artificiales y análisis de conglomerados", Revista Facultad Nacional De Agronomía, vol. 67(2)S2, pp. 812-814, 2014.

[11] A. Gambaro, G. Ares, A. Gimenez, S. Pahor, "Preference mapping of colour of Uruguayan honeys", Journal of Sensory Studies, vol. 22(5), pp. 507-519, 2007.

[12] S. Bogdanov, C. Martin, C. Lullmann, "Harmnized methods of the European Honey Commissionn. International Honey commission. Apidologie, Special Issue”, 2004.

[13] USDA. United States Standards for Grades of Extracted Honey, United States Department of Agriculture, 1985.

[14] R. Fell, "The color grading of honey", American Bee Journal, vol. 118(12), pp. 782-789, 1978.

[15] A. Negueruela, C. Perez-Arquillue, "Color Measurement of Rosemary Honey in the Solid
State by Reflectance Spectroscopy with Black Background", Journal of AOAC International, vol. 83(3), pp. 669-674, 2000.

[16] A. Gomez-Pajuelo, Mieles de España y Portugal. Conocimiento y cata. Ed. Montagud. España, 2004.

[17] A. Lazaridou, C. Biliaderis, N. Bacandritsos, A. Sabatini, "Composition, thermal and rheological behaviour of selected Greek honeys", Journal of Food Engineering, vol. 64(1), pp. 9-21, 2004.

[18] G. Salamanca, Criterios relativos al análisis sensorial de mieles [Online]. 2007, Disponible www.beekeeping.com

[19] S. Aaubert, M. Gonnet, "Mesure de la couleur des miels", Apidologie, vol. 14, pp. 105-118, 1983.

[20] L. Persano, S. Bogdanov, "Determination of honey botanical origin: Problems and issues", Apidologie, vol. 35, pp. S2-S3, 2004.

[21] C. Carvalho, G. Sodré, A. Fonseca, R. Alves, B. Souza, L. Clarton, "Physicochemical characteristics and sensory profile of honey samples from stingless bees (Apidae: Meliponinae) submitted to a dehumidification process", Anais da Academia Brasileira de Ciências, vol. 81(1), pp. 143-149, 2009.

[22] G. Marcazzan, M. Magli, L. Piana, A. Savino, M. Stefano, "Sensory profile research on the main Italian typologies of monofloral honey: possible developments and applications", Journal of Apicultural Research, vol. 53(4), pp. 426- 437, 2014.

[23] A. Soria, M. Gonzalez, C. Lorenzo, I. Martínez, Castroa, J. Sanza, "Characterization of artisanal honeys from Madrid (Central Spain) on the basis of their melissopalynological, physicochemical and volatile composition data", Food Chemistry, vol. 85, pp. 121-130, 2004.

[24] C. Sáinz, C. Gómez, Mieles españolas. Características e identificación mediante el análisis del pollen, E. mundi Prensa, Madrid España, 2000.

[25] B. Debsaka, B. Guzowska-Swider, "Application of artificial neural network in food classification", Analytica Chimica Acta, vol. 705(1-2), pp. 283-291, 2011.

[26] F. Marini, "Artificial neural networks in foods tuff analyses: Trends and perspectives A review", Analytica Chimica Acta, vol. 635, pp. 121-131, 2009.

[27] F. Marini, "Artificial neural networks in foods tuff analyses: Trends and perspectives A 
review", Analytica Chimica Acta, vol. 635, pp. 121-131, 2009.

[28]F.Marini, R. Bucci, R. Magrí, A. Magrí, “Artificial neural networks in chemometrics: History, examples and perspectives.", Microchemical Journal, vol. 88, pp. 178-185, 2008.

[29] F. Marini, A. Magrí, R. Bucci, "Chemometrics and Intelligent Laboratory Systems", vol. 87, pp. 43-49, 2007.

[30] I. Basheer, M. Hajmeer, "Artificial neural networks: fundamentals, computing, design, and application", vol. 43, pp. 3-31, 2000.

[31] H. Álvarez, G. Salamanca, "Caracterización de mieles a través del análisis factorial múltiple y el modelamiento PLS PATH", Alimentos Ciencia e Ingeniería, vol. 16(1), pp. 311-314, 2007.

[32] M. Tenenhaus, J. Pagès, L. Ambroisine, C. Guinot "PLS methodology to study relationships between hedonic judgements and product characteristics", Food Quality and Preference, vol. 16(1), pp. 311-314, 2005.

[33] M. Tenenhaus, V. Vinzi, Y. Chatelin, C. Lauro, "PLS path modelling. Computational Statistics and Data Analysis", vol. 48, pp. 159-205, 2005.

[34] J. Pagés, M. Tenenhaus, "Multiple factor analysis combined with PLS path modelling. Application to the analysis of relationships bet- ween physicochemical variables, sensory profiles and hedonic judgements", Chemometrics and Intelligent Laboratory Systems, vol. 58, pp. 261-273, 2001.

[35] Y. Huang, L. Kangas, B. Rasco, “Applications of artificial neural networks (ANNs) in food science", Critical Reviews Food Science and Nutrition, vol. 47(2), pp. 113-126, 2007.

[36] A. Kouchakzadeh, A. Brati, "Discrimination of Pistachios Varieties with Neural Network using some Physical Characteristic", Int. J. Emerg. Sci, vol. 2(2), pp. 259-262, 2012.

[37] I. Bajwa, M. Choudhary, A study for prediction of minerals in rock images using back propagation neural networks. International conference on advances in space technologies, pp. 185- 189, 2012.

[38] A. Rodríguez, M. Reyes, G. Salamanca, "Clasificación de mieles colombianas mediante sistema de redes neuronales artificiales", Libro de Actas. Congreso Iberoamericano de Ingeniería de Alimentos. Cibia 9. Vol 1. 313322.

[39] K. Anderson, B. Magnuson, M. Tschirgi, B. Smith, "Clasificación de mieles colombianas mediante sistema de redes neuronales artificiales", Journal of agricultural and food chemistry, vol. 47(4), pp. 1568-1575, 1999.

[40] J. Zupan, J. Gasteiger, Neural Networks in Chemistry and Drug Design 2nd ed., Wiley VCH Ed., Weinheim, 1999.

[41] B. Del Brio, M. Serrano, C. Serrano, "Fundamentos de las redes neuronales artificiales: hardware y software.", Scire, vol. 1(1), pp. 104- 124, 1995.

[42] M. Ciappini, M. Di Vito, M. Gatti, A. Calviño, "Development of a Quantitative Descriptive Sensory Honey Analysis: Application to Eucalyptus and Clover Honeys", Advance Journal of Food Science and Technology, vol. 5(7), pp. 829-838, 2013.

[43] G. Gonzalez, C. Lawrence, R. Perez, "Development of a structured sensory analysis honey: Application to artisanal honeys Madrid", Food. Sci. Tech. Int, vol. 16(1), pp. 19-29, 2010.

[44] R. Swanson, C. Lewis, "Premium honeys: response of sensory panelist.", Food Quality and Preference, vol. 3(4), pp. 215-221, 1992.

[45] P. Vit, A. Rodríguez-Malaver, D. Roubik, E. Moreno, B. Almeida Souza, M. Sancho, M. Fernández-Muiño, D. Almeida-Anacleto, L. Marchini, F. Gil, C. Gonzalez, G. Aguilera, B. Nieves, "Expanded parameters to assess the quality of honey from Venezuelan bees (Apis mellifera)", Journal of ApiProduct and ApiMedical Science, vol. 1(3), pp. 72-81, 2009.

[46] B. Sulbarán de Ferrer, G. Ojeda de Rodríguez, J. Peña, J. Martínez, M. Morán, "Mineral content of the honey produced in Zulia state, Venezuela", Archivos Latinoamericanos de Nutrición, vol. 54(3), pp. 346-348, 2004.

[47] L. Piana, L. Persano, A. Bentabol, E. Bruneau, S. Bogdanov, C. Guyot, "Sensory analysis applied to honey: state of the art.", Apidologie, vol. 35, pp. S26-S37, 2004.

[48] IHC (International Honey Commis sion). "Minutes of the IHC meetings: Toledo (1998), Dijon (1999), Celle (2000), Louvain-la-Neuve (2001a), Ate ne (2001b), Celle (2002)", [Online], www.agroscope.admin.ch/imkerei/ index.html?lang=en, 2001.

[49] E. Bruneu, E. Barbier, C. Gallez, L. Guyot, "SAtomesmiels wheel.", Bees C, vol. 77, pp. 16-23, 2000.

[50] G. Montenegro, M. Gómez, R. Pizarro, G. Casaubon, G., R. Peña, "Implementación de un panel sensorial para mieles chilenas.", Ciencia e Investigaciones Agrarias, vol. 35(1), pp. 5158, 2008. 
[51] M. Ciappini, M., M. Di Vito, M. Gatti, A. Cal- viño, "Development of a Quantitative Descriptive Sensory Honey Analysis: Application to Eucalyptus and Clover Honeys", Advance Journal of Food Science and Technology, vol. 5(7), pp. 829-838, 2013.

[52] M. Ciappini, M., M. Di Vito, M. Gatti, A. Cal- viño, "Development of a Quantitative Descriptive Sensory Honey Analysis: Application to Eucalyptus and Clover Honeys", Advance Journal of Food Science and Technology, vol. 5(7), pp. 829-838, 2013.

[53] C. Barrios, Y. Morales, N. Cugnata, F. De Piano, S. Fuselli, M. Maggi, H. Melo, J. Principal, "La apicultura como estrategia de gestión ambiental en la cuenca del embalse Guaremal, municipio Peña, estado Yaracuy, Venezuela", Zootecnia Tropical, vol. 30(3), 269-284, 2012.

[54] U. Kropf, M. Jamnik, J. Bertoncelj, T. Golob. Linear Regression Model of the Ash Mass Fraction and Electrical Conductivity for Slovenian Honey. Food Technol. Biotechnol., vol. 46 (3), pp. 335-340, 2008.
[55] I. Bettar, L. Gonzalez, D. Hernanz, A. Marconi, F. Heredia, A. Terrab. Characterisation of Moroccan Spurge (Euphorbia) honeys by their physicochemical characteristics, mineral contents and colour. Arabian Journal of Chemistry, In press, 2015.

[56] M. Sousa, L. Días, A. Veloso, L. Estevinho, A. Peres, A. Machado. Practical procedure for discriminating monofloral honey with a broad pollen profile variability using an electronic tongue. Talanta, vol. 128, pp. 284-292, 2014.

[57] L. P. Oddo, T. A. Heard, A. Rodríguez-Malaver, R. Perez, M. Fernández, M. Sancho, G. Sesta, L. Lusco, P. Vit. Composition and antioxidant activity of Trigona carbonaria honey from Australia. Journal of Medicinal Food, vol. 11(4), pp. 789-794, 2008.

[58] F. Tornuk, S. Karaman, I. Ozturk, O. S. Toker, B. Tastemur, O. Sagdic, M. Dongan, A. Kayacier. Quality characterization of artisanal and retail Turkish blossom honeys: determination of physicochemical, microbiological, bioactive properties and aroma profile. Industrial Crops and Products, vol. 46, pp. 124-131, 2013. 